\title{
Early versus late pulmonary rehabilitation in chronic obstructive pulmonary disease patients with acute exacerbations: A randomized trial
}

\author{
Puhan, M A ; Spaar, A ; Frey, M ; Turk, A ; Brändli, O ; Ritscher, D ; Achermann, E ; Kaelin, R ; \\ Karrer, W
}

\begin{abstract}
Background: Around the world, the timing of referral of chronic obstructive pulmonary disease (COPD) patients for pulmonary rehabilitation differs from immediately after exacerbation (early) to later on when patients are in a stable state (late). There are no trials comparing the different time points of referral for pulmonary rehabilitation. Objectives: Our aim was to compare the effects of early and late pulmonary rehabilitation on exacerbation rates and health-related quality of life (HRQOL) in COPD patients with exacerbations. Methods: We randomized COPD patients (Global Initiative for Chronic Obstructive Lung Disease stages II-IV) with a recent exacerbation to early (within 2 weeks) or late pulmonary rehabilitation (starting 6 months after randomization and in a stable state). The primary outcome was the exacerbation rate over 18 months, and secondary outcomes included HRQOL and mortality. We used multivariate analyses and an intention-to-treat analysis approach. Results: We randomized 36 patients to pulmonary rehabilitation. On average, patients with early rehabilitation (n $=19$ ) had 2.61 (SD 2.96) exacerbations requiring systemic corticosteroids and/or antibiotics, compared to 2.77 (SD 3.41) in patients with late rehabilitation (adjusted incidence rate ratio $0.83,95 \%$ confidence interval $0.43-1.63 ; \mathrm{p}=0.60)$. Over the 18 -month period, patients with late rehabilitation experienced more dyspnea (difference on Chronic Respiratory Questionnaire dyspnea domain 0.74 and on the Medical Research Council dyspnea scale 0.37), but neither these differences nor any difference in HRQOL domains reached statistical significance. Conclusions: We did not find any statistically significant differences between early and late pulmonary rehabilitation. However, our trial indicates that early rehabilitation may lead to faster recovery of HRQOL after exacerbations compared to rehabilitation later on when patients are in a stable state.
\end{abstract}

DOI: https://doi.org/10.1159/000329884

Posted at the Zurich Open Repository and Archive, University of Zurich

ZORA URL: https://doi.org/10.5167/uzh-61158

Journal Article

Published Version

Originally published at:

Puhan, M A; Spaar, A; Frey, M; Turk, A; Brändli, O; Ritscher, D; Achermann, E; Kaelin, R; Karrer, W (2012). Early versus late pulmonary rehabilitation in chronic obstructive pulmonary disease patients with acute exacerbations: A randomized trial. Respiration, 83(6):499-506.

DOI: https://doi.org/10.1159/000329884 


\title{
Early versus Late Pulmonary Rehabilitation in Chronic Obstructive Pulmonary Disease Patients with Acute Exacerbations: A Randomized Trial
}

\author{
Milo A. Puhan ${ }^{\mathrm{a}, \mathrm{h}} \quad$ Anne Spaar ${ }^{\mathrm{a}} \quad$ Martin Frey $^{\mathrm{b}} \quad$ Alexander Turk $^{\mathrm{c}}$ Otto Brändlic \\ Daniel Ritscher $^{d}$ Eva Achermann ${ }^{\mathrm{e}}$ Rainer Kaelin ${ }^{f}$ Werner Karrer ${ }^{g}$ \\ a Horten Center for Patient-Oriented Research and Knowledge Transfer, University of Zurich, Zurich, \\ ${ }^{b}$ Klinik Barmelweid, Barmelweid, ' Zürcher Höhenklinik Wald, Wald-Faltigberg, 'Lungenpraxis Morgental, \\ Zurich, ${ }^{\mathrm{e} L i m m a t t a l s p i t a l, ~ S c h l i e r e n, ~}{ }^{\mathrm{f}}$ Private practice, Morges, and ${ }^{9}$ Luzerner Hoehenklinik Montana, \\ Crans-Montana, Switzerland; hDepartment of Epidemiology, Johns Hopkins Bloomberg School of Public Health, \\ Baltimore, Md., USA
}

\section{Key Words}

Chronic obstructive pulmonary disease $\cdot$ Rehabilitation •

Exacerbation · Randomized trials • Quality of life

\begin{abstract}
Background: Around the world, the timing of referral of chronic obstructive pulmonary disease (COPD) patients for pulmonary rehabilitation differs from immediately after exacerbation (early) to later on when patients are in a stable state (late). There are no trials comparing the different time points of referral for pulmonary rehabilitation. Objectives: Our aim was to compare the effects of early and late pulmonary rehabilitation on exacerbation rates and health-related quality of life (HRQOL) in COPD patients with exacerbations. Methods: We randomized COPD patients (Global Initiative for Chronic Obstructive Lung Disease stages II-IV) with a recent exacerbation to early (within 2 weeks) or late pulmonary rehabilitation (starting 6 months after randomization and in a stable state). The primary outcome was the exacerbation rate over 18 months, and secondary outcomes included HRQOL and mortality. We used multivariate analyses and an intention-to-treat analysis approach. Results: We randomized 36 patients to pulmonary rehabilitation. On aver-
\end{abstract}

\section{KARGER}

Fax +41613061234 E-Mail karger@karger.ch www.karger.com
(C) 2011 S. Karger AG, Basel

0025-7931/12/0836-0499\$38.00/0

Accessible online at:

www.karger.com/res age, patients with early rehabilitation $(n=19)$ had 2.61 (SD 2.96) exacerbations requiring systemic corticosteroids and/ or antibiotics, compared to 2.77 (SD 3.41) in patients with late rehabilitation (adjusted incidence rate ratio $0.83,95 \%$ confidence interval $0.43-1.63 ; p=0.60$ ). Over the 18 -month period, patients with late rehabilitation experienced more dyspnea (difference on Chronic Respiratory Questionnaire dyspnea domain 0.74 and on the Medical Research Council dyspnea scale 0.37), but neither these differences nor any difference in $\mathrm{HRQOL}$ domains reached statistical significance. Conclusions: We did not find any statistically significant differences between early and late pulmonary rehabilitation. However, our trial indicates that early rehabilitation may lead to faster recovery of HRQOL after exacerbations compared to rehabilitation later on when patients are in a stable state.

Copyright $\odot 2011$ S. Karger AG, Basel

\section{Introduction}

Pulmonary rehabilitation is an effective intervention for patients with chronic obstructive pulmonary disease (COPD) and is recommended by clinical guidelines $[1,2]$. 
There is unequivocal evidence from meta-analyses that pulmonary rehabilitation improves symptoms, healthrelated quality of life (HRQOL) and exercise capacity compared to standard care without rehabilitation [3]. Also, an increasing number of head-to-head trials are informing clinical practice with regard to how to design physical exercise programs that are both feasible and effective for COPD patients $[4,5]$.

Around the world, the timing of referral of COPD patients for pulmonary rehabilitation differs from immediately after exacerbation (early) to later on while the patient is in a stable state (late) [2]. Recent evidence suggests that pulmonary rehabilitation may be equally if not more effective when started immediately after an acute exacerbation [6]. A recently updated Cochrane review identified nine randomized trials comparing rehabilitation and standard care after a COPD exacerbation. The metaanalyses showed similar effects of pulmonary rehabilitation after exacerbations on HRQOL [pooled mean difference in Chronic Respiratory Questionnaire (CRQ) domains between rehabilitation and standard care groups of around 0.9] compared to the effects seen in the metaanalyses of trials that compared rehabilitation and standard care in stable patients (pooled mean difference around 0.9 ), while the effects on exercise capacity appeared to be greater [7]. Even more importantly, the metaanalyses for exacerbations and mortality showed that pulmonary rehabilitation after exacerbation led to a large reduction in the risk of readmission and mortality as compared with standard care.

Reasons for the effects of such early rehabilitation may include the use of a window of opportunity to refer COPD patients for rehabilitation when they are receptive to changing their health behavior. Moreover, patients experiencing exacerbations often have a number of modifiable risk factors for further exacerbations and early death that can be addressed effectively by rehabilitation $[8,9]$. However, it may also just be easier to detect an effect of rehabilitation on hospital admissions and death because patients who have just suffered an exacerbation are at greater risk for exacerbations compared to patients in a stable state. Potential drawbacks of early rehabilitation include the risk of further exacerbations during the rehabilitation that may interrupt the program. Also, patients in an unstable pulmonary condition usually require more supervision compared to patients in a stable state.

While in some countries most patients are referred for (late) pulmonary rehabilitation while in a stable state, in other countries patients are traditionally referred for (early) pulmonary rehabilitation just after an exacerba- tion [2]. There are no head-to-head trials comparing different referral strategies for pulmonary rehabilitation; all the trials in the Cochrane review compared early rehabilitation to standard care. Our aim was to assess whether early rehabilitation after an exacerbation is more effective to reduce exacerbations and to improve HRQOL compared with late pulmonary rehabilitation in patients with severe COPD at risk for exacerbations.

\section{Methods}

Study Design and Patients

We conducted a multicenter, randomized controlled trial in Switzerland. We randomized COPD patients who were treated for an exacerbation to early and late pulmonary rehabilitation using an online central randomization system (www.randomizer.at, University of Graz) that was accessible to site investigators using a password. We used a computerized 'minimization' procedure that avoids chance baseline imbalances in prognostically important variables, which included the modified body mass index, obstruction, dyspnea and exercise capacity (BODE) index (1-4 vs. 5-7 points), in- or outpatient treatment of the acute exacerbation before enrolment, the number of hospitalizations in the previous year $(<2$ and $\geq 2)$ and whether patients had had respiratory rehabilitation in the previous year (yes or no). Using the central randomization and minimization, site investigators could not foresee treatment allocation, which ensured concealment of random allocation.

We included patients with COPD, Global Initiative for Chronic Obstructive Lung Disease stages II-IV, who were 40 years or older and had just undergone treatment of an acute exacerbation in private pulmonary practices and acute care clinics in Switzerland. We defined a COPD exacerbation as a sustained worsening over days to weeks of the patient's symptoms from his or her usual stable state that required in- or outpatient medical treatment. Patients had to have suffered from at least 2 exacerbations in the previous 2 years requiring in- or outpatient care and needed to have been diagnosed with COPD during a stable phase within 3 years before enrolment. The patients needed to be in a medical condition that allowed immediate pulmonary rehabilitation or recovery at home (see exclusion criteria below). Exclusion criteria included hospitalization for other reasons than a COPD exacerbation, long-term noninvasive ventilation (except for continuous pressure ventilation for obstructive sleep apnea, which was allowed) and other lung diseases such as doctor-diagnosed asthma. We excluded patients who were not eligible for either treatment arm because of an impaired level of consciousness, acute confusion, acute changes on the radiograph or electrocardiogram or arterial $\mathrm{pH}<7.35$, orthopedic, rheumatologic, cardiovascular or neurological disorders that inhibit exercise training, gymnastics or guided walking tours, inability to follow patient education in German, French or Italian or mental disorders (e.g. substance abuse, psychosis, dementia). The protocol was approved by all regional ethics committees. All patients were informed about the study both orally and in writing, and they provided written informed consent. 


\section{Pulmonary Rehabilitation}

The 12-week pulmonary rehabilitation programs were identical for both groups and took place in an in- or outpatient rehabilitation center with accreditation from the Swiss Society of Pneumology following the guidelines of the 'Working group for rehabilitation and patient education' of the Swiss Society of Pneumology. The decision about the setting of the rehabilitation was at the discretion of the physician who treated the patient for his/ her exacerbation but we proposed an algorithm to them that considered the severity of disease and the severity of the exacerbation. For patients who had suffered a severe exacerbation (requiring inpatient treatment) and who had a modified BODE score of 5-7, the algorithm proposed a 3 -week inpatient rehabilitation followed by a 9 -week outpatient rehabilitation. For patients with a mild to moderate exacerbation (requiring outpatient treatment) and a modified BODE score of $<5$, we proposed a 12 -week outpatient rehabilitation. For patients with other combinations, we did not propose in- or outpatient rehabilitation specifically. We recommended rehabilitation with 24 exercise sessions but allowed for a range from 18 to 36 exercise sessions considering the diversity of the different programs. The exercise sessions included endurance and strength training as well as calisthenics, as described previously [10]. For patient education, we used the well-established and effective 'Living well with COPD' program [11], which we had previously translated into German and Italian; for French speakers we adapted the Canadian French version. The 'Living well with COPD’ program includes several modules in which patients learn how to use an individual action plan, how to avoid situations and exposures that worsen their symptoms, why and how to use their medication and long-term oxygen, how to exercise in the home environment and how to adapt their daily planning according to their individual abilities. Patients also learned about the importance of smoking abstinence and about the development and consequences of COPD. We conducted extensive pilot testing and training of the patient education material at the study sites and considered feedback to improve the material. With the Swiss version of the 'Living well with COPD' program we also aimed at harmonizing the approach to patient education across rehabilitation centers. However, since some programs already had an established patient education program, we allowed for a stepwise transition to the 'Living well with COPD' program. Nevertheless, we made at least the use of the individual action plan of the 'Living well with COPD' program a prerequisite for participation in the trial.

\section{Outcome Measures}

The primary outcome was the rate of exacerbations during an 18-month follow-up. We only considered exacerbations that required out- or inpatient treatment with systemic corticosteroids and/or antibiotics. The secondary outcomes included HRQOL as measured by the self-administered standardized CRQ $[12,13]$ and the Feeling Thermometer [14], dyspnea as measured by the modified Medical Research Council dyspnea scale and mortality. All of these outcomes have been validated in the languages required here (German, French and Italian) and have seen extensive use in clinical research and practice. We performed the follow-up examinations by mail (questionnaires) and telephone interviews with patients and primary care physicians (exacerbations).

Early versus Late Pulmonary

Rehabilitation in COPD Patients

\section{Sample Size}

To be eligible for our trial, patients needed to have at least one exacerbation per year, as ascertained retrospectively at baseline from patient and physician reports. For the duration of the trial, we expected a higher number of exacerbations because of the prospective ascertainment of exacerbations by regular interview with patients and their general practitioners. Assuming a mean of 3.0 exacerbations requiring medical (in- or outpatient) treatment per patient per year with delayed rehabilitation, we calculated that 118 patients per group will give $80 \%$ power to detect a $20 \%$ reduction to 2.4 admissions per patient per year by immediate rehabilitation. Assuming a dropout rate of $15 \%$, the total sample size increases to 270 . As reported previously, we were not able to recruit that number of patients for various reasons [15]. We introduced many strategies to foster recruitment and also conducted a survey to learn about barriers for recruitment, all of which has been reported previously.

\section{Statistical Analysis}

The main analysis was based on an intention-to-treat approach but we also repeated and reported the analyses including only those patients who finally underwent early or late rehabilitation (per-protocol analysis). We first determined the extent of missing data, which was $<5 \%$ for all outcomes and prognostic variables used for adjustment. Nevertheless, given the limited sample size and in order to avoid selection bias, we used multiple imputation using the 'ice' command of Stata and generated ten data sets. All analyses were based on these data sets using the 'micombine' command of Stata. We compared exacerbation rates between treatment groups using a negative binomial regression model rather than a Poisson regression model since standard deviations were considerably larger than the mean exacerbation rates, indicating overdispersion. We censored patients if they died or were lost to follow-up. We performed the analyses with and without adjusting for stratification variables. In the protocol, we had defined a priori that we would consider early and late rehabilitation to be of equivalent effectiveness if the difference in mean exacerbation rates was $<0.5$ exacerbations per person year. For the comparison of secondary outcomes between randomization and follow-up, we used a generalized estimating equation that accounts for the clustering of repeated measurements $(0,3,6,12$ and 18 months after randomization). This regression analysis gives the average difference between groups over the period of observation. We performed all analyses using Stata for Windows (version 10.1, College Station, Tex., USA).

\section{Results}

We randomized 36 COPD patients to early and late pulmonary rehabilitation after an exacerbation. Participants were elderly patients with a long-standing diagnosis of COPD and severely impaired lung function, and the majority of patients had been admitted for an exacerbation in the 2 years before study enrolment (table 1). Three out of 4 patients had a least one comorbidity, and 
Table 1. Baseline characteristics

\begin{tabular}{|c|c|c|}
\hline & $\begin{array}{l}\text { Early reha- } \\
\text { bilitation } \\
(\mathrm{n}=19)\end{array}$ & $\begin{array}{l}\text { Late reha- } \\
\text { bilitation } \\
(\mathrm{n}=17)\end{array}$ \\
\hline \multicolumn{3}{|l|}{ Demographic characteristics } \\
\hline Age, years & $67.5 \pm 9.8$ & $66.5 \pm 6.2$ \\
\hline Males & $12(63)$ & $9(53)$ \\
\hline Retired & $17(90)$ & $14(88)$ \\
\hline \multicolumn{3}{|l|}{ COPD-specific characteristics } \\
\hline Years since diagnosis & $8.0 \pm 4.8$ & $8.0 \pm 4.6$ \\
\hline Pack years of smoking & $58.6 \pm 34.8$ & $57.6 \pm 21.9$ \\
\hline Current smokers, $\mathrm{n}$ & $4 \pm 21$ & $5 \pm 29$ \\
\hline \multicolumn{3}{|l|}{$\mathrm{FEV}_{1}$ (postbronchodilation) } \\
\hline liters & $1.1 \pm 0.4$ & $1.1 \pm 0.3$ \\
\hline \multicolumn{3}{|l|}{$\mathrm{FEV}_{1}$ (postbronchodilation) } \\
\hline$\%$ predicted & $42.7 \pm 13.5$ & $46.3 \pm 15.9$ \\
\hline FVC, liters & $2.5 \pm 0.9$ & $2.5 \pm 0.6$ \\
\hline $\mathrm{FEV}_{1} / \mathrm{FEV}$ & $0.45 \pm 12.1$ & $0.46 \pm 0.11$ \\
\hline \multicolumn{3}{|l|}{ Patients with $\geq 1$ hospital } \\
\hline admissions in previous 2 years & $13(68)$ & $14(82)$ \\
\hline \multicolumn{3}{|l|}{ Patients on systemic } \\
\hline corticosteroids & $5(26.3)$ & $3(17.7)$ \\
\hline Patients on inhaled corticosteroids & $15(79.0)$ & $17(100)$ \\
\hline \multicolumn{3}{|c|}{ Patients on long-acting } \\
\hline bronchodilators & $17(89.4)$ & $17(100)$ \\
\hline \multicolumn{3}{|l|}{ Comorbidities } \\
\hline$\geq 1$ comorbidity & $14(74)$ & $14(82)$ \\
\hline Cardiovascular & $8(42)$ & $12(71)$ \\
\hline Endocrine & $1(5)$ & 0 \\
\hline Musculoskeletal & $1(5)$ & $1(6)$ \\
\hline Other & $8(42)$ & $3(18)$ \\
\hline \multicolumn{3}{|l|}{ Patient-reported outcomes } \\
\hline CRQ dyspnea & $3.61 \pm 1.78$ & $3.08 \pm 1.08$ \\
\hline CRQ fatigue & $3.64 \pm 1.15$ & $3.57 \pm 1.18$ \\
\hline CRQ emotional function & $3.97 \pm 1.29$ & $3.85 \pm 1.15$ \\
\hline CRQ mastery & $4.04 \pm 1.71$ & $3.88 \pm 1.35$ \\
\hline MRC dyspnea scale & $2.26 \pm 1.24$ & $2.41 \pm 0.71$ \\
\hline Feeling Thermometer & $50.4 \pm 19.3$ & $47.5 \pm 12.1$ \\
\hline \multicolumn{3}{|l|}{ Prognostic assessment } \\
\hline ADO index & $4.8 \pm 1.8$ & $4.5 \pm 1.0$ \\
\hline Risk of 3-year death, \% & $30.6 \pm 13.4$ & $27.9 \pm 7.1$ \\
\hline
\end{tabular}

Values are means \pm SD or numbers (percentages), as appropriate. All lung function values refer to measurements during the stable state within 3 years before randomization. $\mathrm{MRC}=$ Medical Research Council; ADO = age, dyspnea and airflow obstruction.

patients randomized to late rehabilitation had a higher prevalence of cardiovascular comorbidity. Patient-reported outcomes indicated severely impaired HRQOL and high levels of dyspnea, and on average, patients were at high risk of death within 3 years (table 1).
Four patients in each group did not start the pulmonary rehabilitation (fig. 1). In the early rehabilitation group, 1 patient died before the beginning of the pulmonary rehabilitation, 1 patient had another exacerbation and no longer wanted to participate in a rehabilitation program, 1 patient declined rehabilitation after randomization because he did not feel to be in a physical condition for physical exercise and 1 patient could not be contacted anymore by the research team nor the treating physician. In the late rehabilitation group, 3 patients no longer wanted to follow a rehabilitation program, and for 1 patient the director of the rehabilitation program considered the patient to be ineligible for the program for unspecified reasons. Overall, 15 (79\%) and 13 patients (76\%) began the early and late rehabilitation, respectively. Five patients with early rehabilitation followed an inpatient program first, which included physical exercise 5 times per week for 3 weeks and the patient education program. However, 3 of them did not follow a supervised outpatient follow-up exercise program and were discharged from the inpatient program with a plan for home-based exercise. Three patients with early rehabilitation had to interrupt or stop the program because of a re-exacerbation. Of the late rehabilitation group, 3 patients nonetheless undertook early rehabilitation; 1 patient decided to stop his program early and 1 patient participating in inpatient rehabilitation did not follow the outpatient follow-up program. In each group, 2 patients died during the follow-up of 18 months.

On average, patients with early rehabilitation suffered from 2.61 (SD 2.96) exacerbations that required treatment with systemic corticosteroids and/or antibiotics over the 18-month period, or 1.93 exacerbations per person year. In the late rehabilitation group, the mean number of exacerbations per patient was 2.77 (SD 3.41), which is 1.89 exacerbations per person year. In the intention-totreat approach, the incidence risk ratio for exacerbations for the comparison of early versus late rehabilitation was 0.96 [95\% confidence interval (CI) $0.48-1.94 ; \mathrm{p}=0.91$ ] without and $0.83(95 \% \mathrm{CI} 0.43-1.63 ; \mathrm{p}=0.60)$ with adjustment for the modified BODE score, in- or outpatient treatment of the acute exacerbation before enrolment, the number of hospitalizations and pulmonary rehabilitation in the previous year. The per-protocol analysis, which only considered patients who underwent early or late rehabilitation, showed an incidence risk ratio for exacerbations of 0.94 (95\% CI 0.52-2.24; $\mathrm{p}=0.89$ ) without and 1.07 (95\% CI $0.52-2.24 ; \mathrm{p}=0.85)$ with adjustment for stratification variables. 
Fig. 1. Study flow: 36 COPD patients randomized to early and late pulmonary rehabilitation.

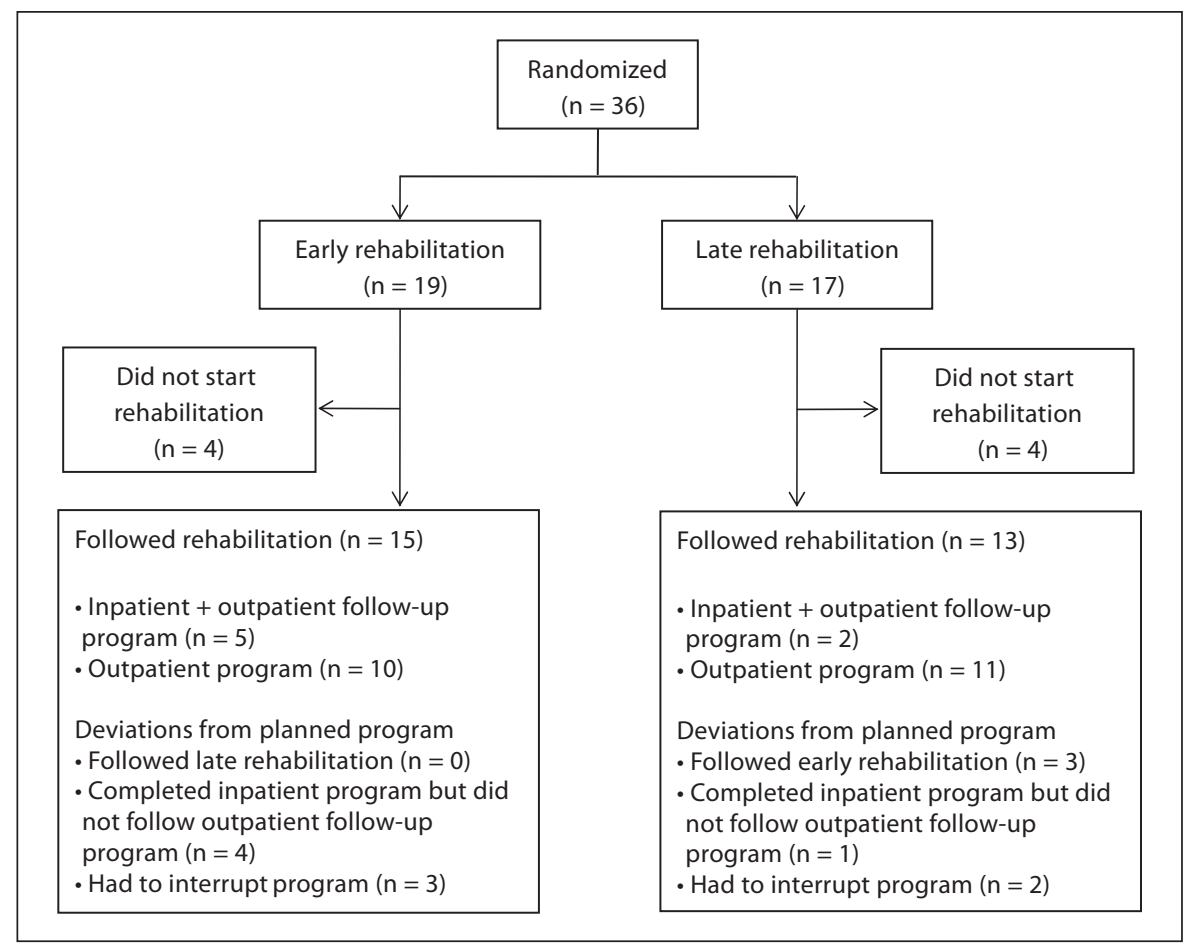

Table 2. Patient-reported outcomes after 6, 12 and 18 months

\begin{tabular}{|c|c|c|c|c|c|c|}
\hline & \multicolumn{6}{|c|}{ Difference between early and late rehabilitation } \\
\hline & at 6 months & $\mathrm{p}$ value & at 12 months & $\mathrm{p}$ value & at 18 months & $\mathrm{p}$ value \\
\hline CRQ dyspnea & $-0.83(-1.95$ to 0.29$)$ & 0.14 & $-0.38(1.65-0.89)$ & 0.54 & $-0.51(-1.77$ to 0.74$)$ & 0.41 \\
\hline CRQ fatigue & $-0.44(-1.26$ to 0.39$)$ & 0.29 & $-0.26(-1.37$ to 0.85$)$ & 0.64 & $-0.54(-1.65$ to 0.57$)$ & 0.33 \\
\hline CRQ emotional function & $-0.22(-1.10$ to 0.67$)$ & 0.62 & $0.21(-0.91$ to 1.34$)$ & 0.70 & $-0.50(-1.51$ to 0.51$)$ & 0.32 \\
\hline CRQ mastery & $-0.50(-1.45$ to 0.44$)$ & 0.28 & $0.53(-0.70$ to 1.76$)$ & 0.39 & $-0.27(-1.13$ to 0.59$)$ & 0.52 \\
\hline MRC dyspnea scale & $0.83(0.10-1.57)$ & 0.028 & $0.63(-0.22$ to 1.48$)$ & 0.14 & $0.27(-0.45$ to 1.00$)$ & 0.45 \\
\hline Feeling Thermometer & $-9.68(-24.70$ to 5.34$)$ & 0.20 & $1.40(-12.00$ to 14.79$)$ & 0.83 & $-6.79(-23.02$ to 9.44$)$ & 0.40 \\
\hline
\end{tabular}

All comparisons were adjusted for baseline values of the outcome, age and $\mathrm{FEV}_{1}$. Values in parentheses represent $95 \% \mathrm{CIs}$. MRC $=$ Medical Research Council.

Figure 2 and table 2 show the patient-reported outcomes over the 18-month follow-up. Dyspnea as measured by the CRQ improved rapidly in the early rehabilitation group and less so in the late rehabilitation group. Between months 6 and 12, dyspnea remained stable in the early rehabilitation group, whereas there was a further improvement in the late rehabilitation group. Over the entire 18-month period, the average difference between the groups was $-0.72(95 \% \mathrm{CI}-1.79$ to 0.32 ; $\mathrm{p}=$ 0.17 ), favoring the early rehabilitation group although not statistically significantly so. We observed a similar pattern for the other CRQ domains, although betweengroup differences were smaller and only present for the first 6 months of follow-up. The Medical Research Council dyspnea scale score closely resembles the result for the CRQ dyspnea domain, but again the differences between groups did not reach statistical significance over the entire study period. Only at 6 months did patients with early rehabilitation have significantly less dyspnea. Finally, the Feeling Thermometer showed dif- 


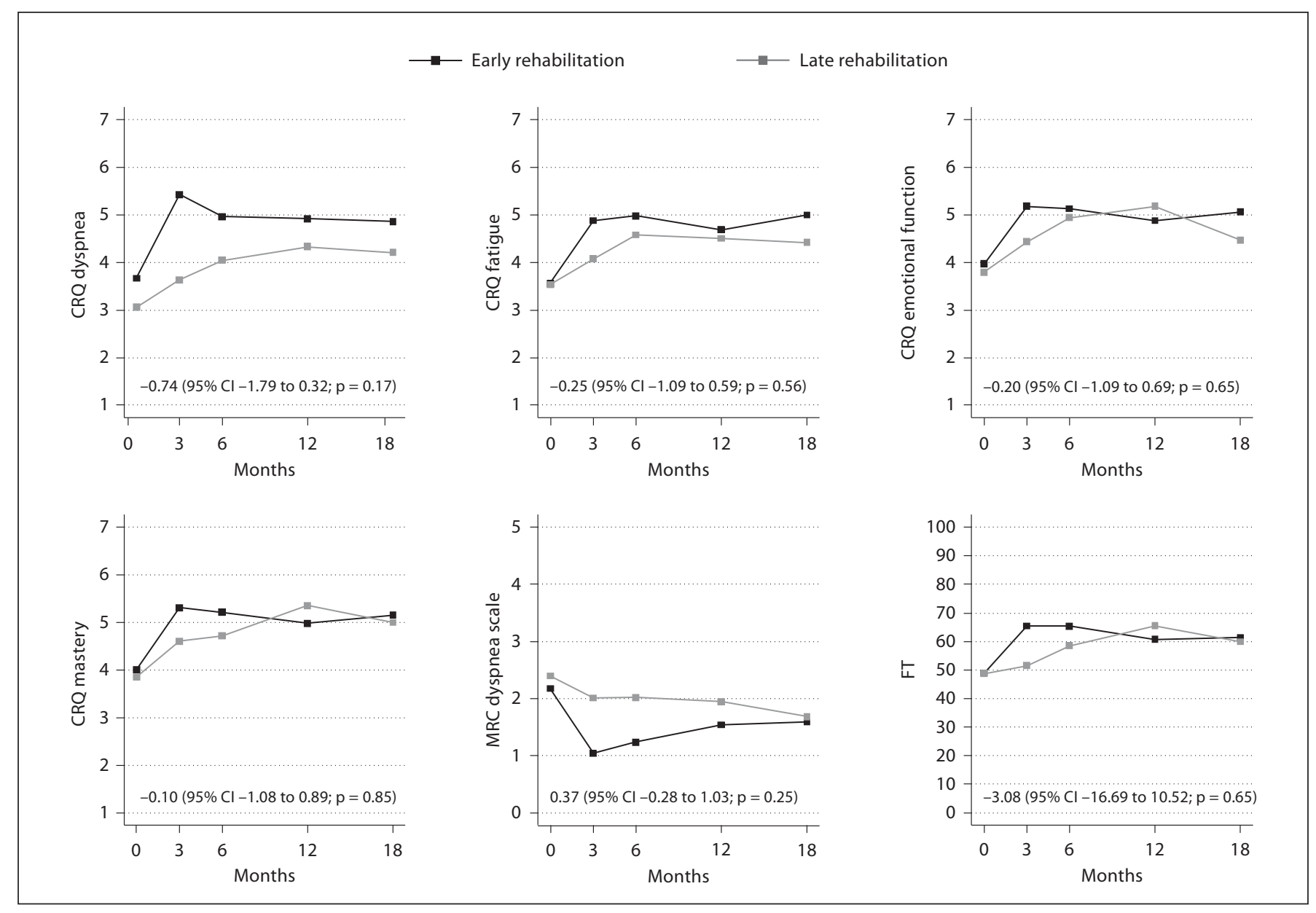

Fig. 2. Patient-reported outcomes over the 18-month follow-up. MRC = Medical Research Council; FT = Feeling Thermometer.

ferences between groups for the first 6 months, whereas the health status of the two groups was similar between months 6 and 18 .

\section{Discussion}

We did not detect an effect of the timing of pulmonary rehabilitation on exacerbation rates in patients with severe COPD. Exacerbation rates of patients undergoing early and late rehabilitation were similar and well below our predefined threshold for a clinically relevant difference. However, patients with early rehabilitation had consistently greater benefits in terms of symptoms and HRQOL over the entire 18-month follow-up, although the differences compared to patients with late rehabilitation did not reach statistical significance.
Exacerbations and readmissions are among the most relevant problems that need to be addressed to improve COPD patient care. Exacerbations have detrimental effects on the patient's health status and prognosis and the cost implications are enormous [16-20]. Unfortunately, most COPD research focuses on the acute treatment of exacerbations or on the long-term management of COPD. Unlike for other chronic diseases, there is almost no evidence or ongoing research on the most effective discharge and postexacerbation management for COPD patients to accelerate recovery from exacerbations and to reduce the risk of more exacerbations and admissions. The recent Cochrane review on rehabilitation after COPD exacerbations indicated that such a multidisciplinary approach that addresses several risk factors for readmissions is effective to reduce readmissions [7]. 
Given the strong evidence for the effectiveness of pulmonary rehabilitation in stable COPD [7] and after an exacerbation [7], we considered it unethical to randomize patients to early rehabilitation and standard care (no rehabilitation). However, we were still interested in observing the effects of early rehabilitation on recovery from exacerbations and for this reason we chose to offer late rehabilitation after an arguably long period of 6 months. Our trial indicated a trend towards accelerated recovery from an exacerbation if patients are referred for early rehabilitation. Patient-reported outcomes showed higher HRQOL for patients with early rehabilitation, and differences exceeded the minimal important difference for some CRQ domain scores (0.5 units) and the Feeling Thermometer (8 units) during the first 6 months, but these results did not reach statistical significance. We did not observe any difference in exacerbation rates between groups, neither from a clinical (difference of $<0.5$ exacerbations per person year as prespecified) nor from a statistical perspective. Finally, we found no indication that either the early or late rehabilitation was less safe.

Our sample size was clearly too small for a definitive answer, but the size of our trial was comparable to those included in the Cochrane review, where the median number of patients included in the nine trials was 42 . As evidence accumulates, it will become clearer whether the timing of referral for pulmonary rehabilitation is critical or, if not, whether the most important decision is to refer patients with severe COPD at risk for exacerbations for pulmonary rehabilitation.

There are also reasons against early rehabilitation, with the most important probably being that patients may have to interrupt the rehabilitation because they experience a re-exacerbation or that they cannot even start the rehabilitation program [2]. In our trial, 3 patients interrupted their early rehabilitation and 4 patients did not start it. Also, if an inpatient rehabilitation program is available, it is more likely that a patient with severe COPD who has suffered from a severe exacerbation will be referred for inpatient rehabilitation. While patients derive great benefit from inpatient rehabilitation, during which they are supervised more closely and compliance is better than in outpatient programs, the transition back to the home environment and to an outpatient follow-up program is often difficult. In our trial, 4 out of 7 patients (in both groups) who undertook an inpatient program did not enroll in an outpatient follow-up program. Thus, although these patients received intense rehabilitation, it is probably more difficult to incorporate regular physical exercise into the patients' everyday life. On the other hand, for patients not referred for early rehabilitation, it is often difficult to convince them to undergo rehabilitation once their health state has stabilized again, so that the overall proportion of patients enrolling in pulmonary rehabilitation might be smaller.

We encountered a number of challenges in our trial. As reported previously [15], recruitment was difficult so that we only randomized $15 \%$ of the planned sample size. As a consequence, the precision for the effect estimates is low. Also, the Swiss health care system suffers, as many others do, from insufficient links between primary care, acute care hospitals and rehabilitation. This is reflected by the difficulty of organizing an outpatient follow-up program when patients need a transition from the inpatient program to implementation of physical exercise in their daily life. It is challenging to ensure continuity of care across these settings, which is so important for effective COPD care. Nevertheless, we believe that the strengths of our trial include the delivery of rehabilitation programs that are effective if patients follow them, a study protocol with attention to methodological details to ensure internal validity and finally the multicentric nature of our trial.

In conclusion, early pulmonary rehabilitation of patients with severe COPD who had suffered from an exacerbation did not reduce exacerbation rates compared with late rehabilitation. However, our trial indicates that postexacerbation management, including pulmonary rehabilitation, may accelerate the recovery from exacerbations.

\section{Acknowledgements}

We thank all the physicians and physiotherapists who collaborated on this study. A special thanks goes to Ursula Schafroth at the study coordinating center.

\section{Financial Disclosure and Conflicts of Interest}

We thank the following funding bodies for their generous financial support: the Swiss Lung League, the Lung Leagues of the cantons of Aargau, Grisons, Lucerne, Nidwalden, Solothurn, Thurgau, Valais, Vaud and Zurich, the Klinik Barmelweid, the 4 clinics of Crans-Montana (Quadrimed), the Höhenkliniken of Zurich as well as Astra Zeneca Switzerland. All these funding bodies provided unrestricted grants to the Swiss Study on Pulmonary Rehabilitation after Exacerbation study group. They did not have any influence on the study design, execution, analysis or reporting of the Swiss Study on Pulmonary Rehabilitation after Exacerbation. 


\section{References}

1 Celli BR, MacNee W: Standards for the diagnosis and treatment of patients with COPD: a summary of the ATS/ERS position paper. Eur Respir J 2004;23:932-946.

2 Nici L, Donner C, Wouters E, Zuwallack R, Ambrosino N, Bourbeau J, Carone M, Celli B, Engelen M, Fahy B, et al: American Thoracic Society/European Respiratory Society statement on pulmonary rehabilitation. Am J Respir Crit Care Med 2006;173:1390-1413.

3 Lacasse Y, Goldstein R, Lasserson TJ, Martin S: Pulmonary rehabilitation for chronic obstructive pulmonary disease. Cochrane Database Syst Rev 2006;4:CD003793.

4 Beauchamp MK, Nonoyama M, Goldstein RS, Hill K, Dolmage TE, Mathur S, Brooks $\mathrm{D}$ : Interval versus continuous training in individuals with chronic obstructive pulmonary disease - a systematic review. Thorax 2010;65:157-164.

5 Puhan MA, Schunemann HJ, Frey M, Scharplatz M, Bachmann LM: How should COPD patients exercise during respiratory rehabilitation? Comparison of exercise modalities and intensities to treat skeletal muscle dysfunction. Thorax 2005;60:367-375.

6 Osthoff M, Leuppi JD: Management of chronic obstructive pulmonary disease patients after hospitalization for acute exacerbation. Respiration 2010;79:255-261.

7 Puhan MA, Gimeno-Santos E, Scharplatz M, Troosters T, Walters EH, Steurer J: Pulmonary rehabilitation following exacerbations of chronic obstructive pulmonary disease. Cochrane Database Syst Rev 2009; 1:CD005305.
8 Garcia-Aymerich J, Barreiro E, Farrero E, Marrades RM, Morera J, Anto JM: Patients hospitalized for COPD have a high prevalence of modifiable risk factors for exacerbation (EFRAM study). Eur Respir J 2000;16: 1037-1042.

9 Puhan MA, Garcia-Aymerich J, Frey M, ter Riet G, Anto JM, Agusti AG, Gomez FP, Rodriguez-Roisin R, Moons KG, Kessels AG, et al: Expansion of the prognostic assessment of patients with chronic obstructive pulmonary disease: the updated BODE index and the ADO index. Lancet 2009;374:704-711.

10 Puhan MA, Busching G, Schunemann HJ, VanOort E, Zaugg C, Frey M: Interval versus continuous high-intensity exercise in chronic obstructive pulmonary disease: a randomized trial. Ann Intern Med 2006;145:816825.

11 Bourbeau J, Julien M, Maltais F, Rouleau M, Beaupre A, Begin R, Renzi P, Nault D, Borycki E, Schwartzman K, et al: Reduction of hospital utilization in patients with chronic obstructive pulmonary disease: a diseasespecific self-management intervention. Arch Intern Med 2003;163:585-591.

12 Puhan MA, Behnke M, Frey M, Grueter T, Brandli O, Lichtenschopf A, Guyatt GH, Schunemann HJ: Self-administration and interviewer-administration of the German Chronic Respiratory Questionnaire: instrument development and assessment of validity and reliability in two randomised studies. Health Qual Life Outcomes 2004;2:1.

13 Puhan MA, Behnke M, Laschke M, Lichtenschopf A, Brandli O, Guyatt GH, Schunemann HJ: Self-administration and standardisation of the chronic respiratory questionnaire: a randomised trial in three German-speaking countries. Respir Med 2004. 98:342-350.
14 Puhan MA, Behnke M, Devereaux PJ, Montori VM, Braendli O, Frey M, Schunemann HJ: Measurement of agreement on healthrelated quality of life changes in response to respiratory rehabilitation by patients and physicians - a prospective study. Respir Med 2004;98:1195-1202.

15 Spaar A, Frey M, Turk A, Karrer W, Puhan MA: Recruitment barriers in a randomized controlled trial from the physicians' perspective: a postal survey. BMC Med Res Methodol 2009;9:14.

16 Garcia-Aymerich J, Farrero E, Felez MA, Izquierdo J, Marrades RM, Anto JM: Risk factors of readmission to hospital for a COPD exacerbation: a prospective study. Thorax 2003;58:100-105

17 Groenewegen KH, Schols AMWJ, Wouters EFM: Mortality and mortality-related factors after hospitalization for acute exacerbation of COPD. Chest 2003;124:459-467.

18 Seemungal T, Donaldson G, Paul E, Bestall J, Jeffries D, Wedzicha J: Effect of exacerbation on quality of life in patients with chronic obstructive pulmonary disease. Am J Respir Crit Care Med 1998;157:1418-1422.

19 Roberts CM, Barnes S, Lowe D, Pearson MG: Evidence for a link between mortality in acute COPD and hospital type and resources. Thorax 2003;58:947-949.

20 Roberts CM, Ryland I, Lowe D, Kelly Y, Bucknall CE, Pearson MG: Audit of acute admissions of COPD: standards of care and management in the hospital setting. Eur Respir J 2001;17:343-349. 